\title{
The role of noise and advection in absorbing state phase transi- tions
}

\author{
C. Barrett-Freeman, M. R. Evans, D. Marenduzzo, J. Tailleur
}

SUPA, School of Physics and Astronomy, University of Edinburgh, Mayfield Road, Edinburgh EH9 $3 J Z$

PACS 64.60.-i - General studies of phase transitions

PACS 05.70.Ln - Nonequilibrium and irreversible thermodynamics

\begin{abstract}
We study the effect of advection and noise on the field theory for directed percolation (DP). We show that even a very small advective velocity is enough to change the universality class of the dynamic phase transition. When the noise is taken to be proportional to the square root of the population density, we find an additional nonequilibrium "spinodal" line separating a region where an exponentially decreasing density is metastable, from another one in which it is unstable. If the noise is instead linear in the density, the phase diagram changes dramatically both quantitatively and qualitatively, and the spinodal line becomes a true phase boundary. We briefly discuss possible applications of our results to microbial sedimentation and population dynamics in rivers.
\end{abstract}

The concept of universality classes has been employed with much success in equilibrium statistical physics, gathéring myriad phase transitions into a handful of classes. The effort to extend and apply this concept to nonequilibrium systems is still ongoing, and is one of the foremost challenges of statistical physics. Probably the widest and best characterised nonequilibrium universality class to date is that of directed percolation (DP) 1, a system oftien described by the following Langevin equation [2]:

$$
\partial_{t} \rho=D \partial_{x x} \rho+a \rho-b \rho^{2}+\sqrt{\rho} \eta
$$

Here, $\rho=\rho(x, t)$ is a density, $D$ a diffusion coefficient, $a>0$ a "growth rate", $b>0$ a saturation constant, and $\eta(x, t)$ is Gaussian white noise of unit variance.

DP is an archetypal nonequilibrium phase transition, which takes an active phase, where the fluctuating density is non-zero, continuously into an absorbing one with zero density. It succesfully describes systems as diverse as chemical reaction-diffusion processes, epidemic spreading, percolation through porous media, growing microbial population and branching-annihilating random walks 3. This broadness is now expressed by the "DP conjecture", stating that all systems exhibiting a continuous transition into a unique absorbing state, characterised by a onecomponent order parameter, and not showing any extra symmetries or conservation laws, belong to the DP universality class 2,4 . The DP conjecture has proven extremely robust to changes in the microscopic dynamics.
This brings us to the subject of this article: what is the fate of the DP universality class in the presence of advection?

One might expect that the addition of an advection term to (11) could be transformed away by a Galilean transformation [5,6] thus rendering it irrelevant. This is however not true in the presence of fixed boundaries. For most equilibrium systems boundaries are not expected to affect the critical behaviour, however in a nonequilibrium system boundaries are known to play a crucial role in determining phase transitions [7]. To illustrate this and gain insight into our question, we consider the effect of advection on the noiseless limit of Eq. 1, which is nothing but the celebrated Fisher-Kolmogoroff (F-KPP) equation [5]. It clearly admits two steady state solutions $(\rho=0, \rho=a / b)$ and exhibits the well-known Fisher Waves: wavefronts emerge from portions of the system in the high density state $\rho=a / b$ and propagate into empty regions with velocity $v_{f}=2 \sqrt{D a}[5$. It has recently been shown $[8,9]$ that in the presence of boundaries, and upon addition of an advection term $v \partial_{x} \rho$, F-KPP exhibits a new lowdensity steady-state: an exponential phase in which the density profile decays exponentially away from the boundary. The competition between the advancing Fisher wave and the advection term triggers a novel discontinuous nonequilibrium phase transition separating this low-density phase $\left(v>v_{f}\right)$ from the high-density one $\left(v<v_{f}\right)$, in which $\rho \simeq a / b$ throughout the system. Restoring noise 


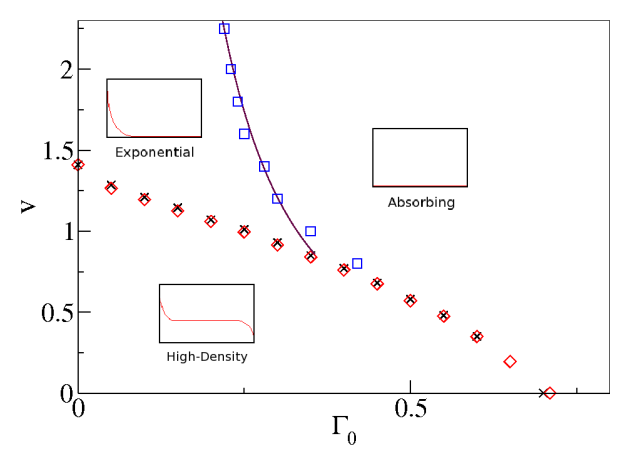

Figure 1: Phase boundary for $a=0.5$ and $\eta \propto \sqrt{\rho}$. The critical points found via the Dickman and Dornic et al. algorithms are shown as black crosses and red diamonds, respectively. Both methods agree up to an accuracy of about $1 \%$. The solid maroon line correspond to the theoretical prediction (see below) for the spinodal line whereas the blue squares correspond to its numerical counterpart (see below).

and returning to DP, it is natural to question the robustness of the exponential profile. Indeed, we now have two candidates for the low-density phase: the exponential one or the usual absorbing state of DP.

In this work, we show that in the presence of advection, the low-density phase is always absorbed at long times whereas the fate of the exponential state depends on the strength of the noise; it is never seen at large noise but is metastable below a certain threshold. The role played by the noise in this dynamical transition is crucial. We explore this issue further by considering noise proportional to the density rather than to its square root and show that this transforms the dynamical transition into a true phase transition. Most importantly, we find that the addition of an advective term to DP, however small, always changes the nature of the transition, rendering it discontinuous. In other words, advection is a relevant perturbation in renormalization group jargon, in a similar way that, for instance, a magnetic field is a relevant perturbation in an Ising model in equilibrium statistical mechanics.

Our starting point is the following extension of Eq. (1)

$$
\partial_{t} \rho=D \partial_{x x} \rho+v \partial_{x} \rho+a \rho-b \rho^{2}+\Gamma_{0} g(\rho) \eta
$$

Our study is entirely carried out in one dimension; extension to higher dimensions would be interesting but, since the advection term can be extended in various ways, we leave this for further study. We consider two different kinds of nonequilibrium noise distinguished by the functional form of $g(\rho)$. In the first part of the article, we choose $g(\rho)=\sqrt{\rho(x, t)}$, as in conventional DP (see Eq. (1)), and refer to this as "square root" noise. Such noise typically arises from fluctuations among individuals in a finite population of average density $\rho(x, t)$. In the second case, that we address toward the end of the paper, we consider a "linear" noise, defined by $g(\rho)=\rho(x, t)$, which is usually met in the context of population dynamics to model fluctuating environments [10]. This kind of noise is sometimes called "multiplicative" in the literature [3, but we use this terminology here to describe generic dependence of the noise on the density. Once $g(\rho)$ is chosen, $\Gamma_{0}$ is a parameter used to scale the strength of the noise relative to the other terms. Throughout the paper, we impose 'no-flux' boundary conditions at the top and bottom of the system 1 , whose size is $L$. As is usual for semi-infinite systems, we first take the large time limit before sending the system size to infinity.

To obtain our numerical results for the square root noise, we used two different algorithms. The main technical difficulty is to ensure that, upon time discretization of Eq. (2), the multiplicative noise does not lead to unphysical negative densities when $\rho$ is small (which is the case, for instance, when we are close to the critical point of DP at $v=0)$. The first method we use was originally proposed by Dickman [11, and entails a symmetrical truncation of the noise coupled with discretization of the density such that $\Gamma_{0 \sqrt{\rho}} \eta$ never exceeds $-\rho(x, t)$. The second is an improved integration scheme proposed by Dornic et al. in [12. Instead of drawing a random Gaussian number at each $x$ to be later on multiplied by $\Gamma_{0} \sqrt{\rho(x, t)}$, this algorithm draws directly from the probability distribution function which solves the Fokker-Planck equation associated with the linear part of the local Langevin equation at each $x$ (see Ref. 12 for details). After this, the deterministic non-linear part of the equation is evolved via a finite difference scheme. In this case, the density is never negative by construction and numerical problems are avoided. We checked that both schemes yield very similar results. In all the simulations presented in this article, we use $a=0.5, b=1$ and $D=1$. Typical integration parameters with Dickman's algorithm are $d x=0.1, d t=0.001$, $L=1000$ whilst varying $v$ and $\Gamma_{0}$. For the Dornic et al. algorithm, whose generalisation to an advection term is quite straightforward, we could choose larger $d x$ up to 0.5 and $d t$ up to 0.05 , considerably reducing simulation time.

First, we map out the phase diagram in the $\Gamma_{0}-v$ parameter plane (see Fig. 1). A full line of critical points $v=v_{c}\left(\Gamma_{0}\right)$ now links the two limiting cases of DP $(v=0)$ and F-KPP with advection $\left(\Gamma_{0}=0\right)$. For small $\Gamma_{0}$ and $v$, we observe a high-density phase, where the total mass $M$ of the system is extensive with system size, whereas the rest of the phase diagram is composed of low-density regions (whether the exponential profile or the absorbing state) where $M / L \rightarrow 0$ as $L \rightarrow \infty$. The absorbing state can be accessed via fluctuations and is thus favoured by larger values of $\Gamma_{0}$. As a consequence, the critical velocity $v_{c}\left(\Gamma_{0}\right)$, above which the high density phase is not observed, is a decreasing function of noise strength.

Interestingly, long time simulations of the system show that its dynamical behaviour in the low-density phase is not uniform. The exponential phase is long-lived for small $\Gamma_{0}$ and we refer to this regime as the 'low noise' case. While holding the velocity fixed, increasing $\Gamma_{0}$ has the

\footnotetext{
${ }^{1}$ i.e, we take $D \partial_{x} \rho+v \rho=0$ at $x=0, L$
} 


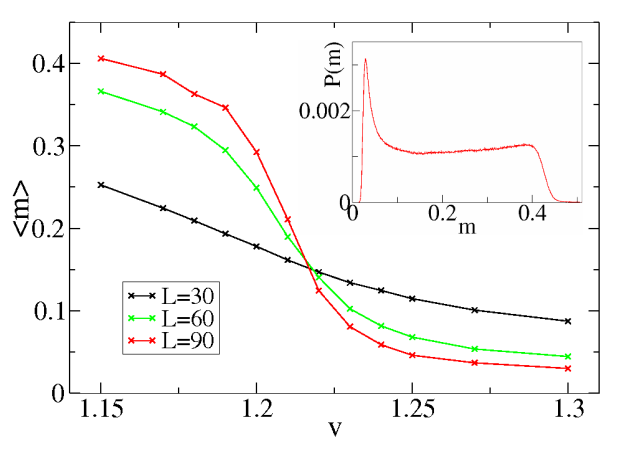

Figure 2: Plots of the average order parameter $\langle m\rangle$ as a function of $v$, for $\Gamma_{0}=0.10$ and for three different system sizes (see legend). The presence of a crossing of the order parameter curves indicates that the transition is discontinuous. Inset: probability distribution of the order parameter, for $v=v_{c} \simeq 1.21$ and $L=100$.

effect of decreasing the average lifetime of the exponential state, until we can barely see it; this is the beginning of the 'strong noise' case. As we shall see, this threshold of $\Gamma_{0}$ corresponds to a (nonequilibrium) spinodal line which separates the two dynamical regimes (See Fig. 1). We now explain how the critical points were obtained and discuss the nature of the phase transition, detailing in particular the differences between the low and strong noise regimes.

In order to identify the critical points we define the order parameter $m=M / L$, where $M$ is the total mass in the system. We compute the average of $m$ in the quasistationary state 2 over a number of different simulations, and then plot this average as a function of $v$, for fixed $\Gamma_{0}$. For finite systems, the crossover between low- and highdensity phases sharpen when the system size is increased, but the order parameter curves intersect at a well-defined non-zero value of $v$ (see Fig. 2 and 3 ), which we take as the critical velocity $v_{c}$. This scenario is indicative of a discontinuous transition: in the thermodynamic limit, as $v \nearrow v_{c}$ the density in the stead state approaches a non-zero value, whereas for $v \searrow v_{c}$ it is strictly zero. Thus, for all nonzero values of $v$, there will be a discontinuous "jump" at the transition point; the system no longer belongs to the DP universality class.

Whereas the nature of the transition differs from that of DP, it is also different from the one of the noiseless case $\left(\Gamma_{0}=0\right)$ discussed in the introduction. In the absence of noise, a band structure is formed at criticality and a stationary front separates high- and low-density regions in the steady state. In the noisy case considered here, the transition is in general different. Even the low noise regime, while still bearing some resemblance to the noiseless case, shows qualitatively distinct behaviour. This last point can be appreciated by looking at the quasistation-

\footnotetext{
${ }^{2}$ This is the steady state of the probability distribution conditioned on survival.
}

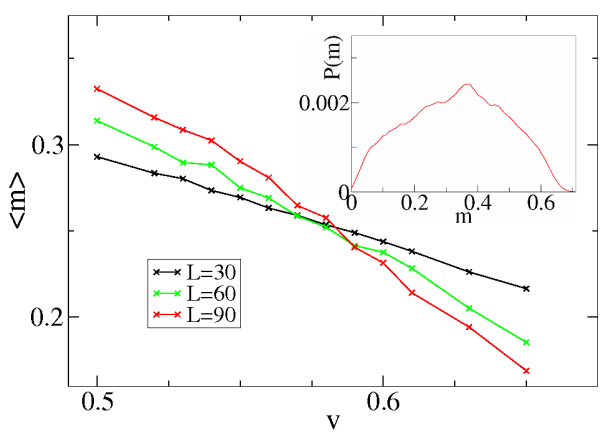

Figure 3: Plots of the average order parameter $\langle m\rangle$ as a function of $v$, for $\Gamma_{0}=0.50$ and for three different system sizes (see legend). The presence of a crossing of the order parameter curves indicates that the transition is discontinuous. Inset: probability distribution of the order parameter, for $v=v_{c} \simeq 0.58$ and $L=100$.

ary probability distribution of the average density of the system at criticality, which is shown in the inset of Fig. 2. This distribution is composed of an approximately flat part coexisting with a peak close to zero. The physical interpretation is that in the low noise regime, the band is still present but the front, no longer stationary, now performs a random walk and occasionally gets stuck in the exponential profile. We checked numerically that the roaming band indeed performs a random walk by measuring the variance of the order parameter as a function of time (data not shown). The order parameter distribution is linked, in equilibrium statistical mechanics, to the shape of the free energy. In the case of a standard discontinuous transition, close to criticality, there would be two coexisting free energy minima which result in two peaks in the probability distribution of the order parameter. In our case however, we have coexistence between one peak corresponding to the exponential profile and a flat piece in which all values of the total mass are essentially equiprobable. The latter piece of the distribution corresponds to the high-density phase, in which the average of the order parameter thus scales with the system size.

The transition into the low-density, large noise regime is quite different and, as might be expected, is reminiscent of what happens in DP. In this regime, there is no longer any front, or band structure, due to the large fluctuations, and the system now uniformly collapses into the absorbing state. The numerics in this region are more difficult. Since we are working with finite size systems, when $v \lesssim v_{c}$ the system is occasionally absorbed, even though it properly belongs to the high-density phase. To overcome this difficulty, we use PERM (Pruning and Enrichment Rosenbluth Method, see [13. for a recent review) for simulating the quasistationary state. Despite the apparent similarity with DP, the transition is still discontinuous, as can be seen in Fig. 3. However, the order parameter distribu- 
tion at criticality also differs from the low noise case (see the inset of Fig. 3). First, the peak corresponding to the exponential phase has disappeared. Then, the flat part in the distribution is now replaced by a broad peak at a finite value. For completeness, the quasistationary distribution, obtained using PERM, should be complemented by a delta function at $\langle\rho\rangle=0$. Therefore this case is closer to a standard discontinuous transition, with two competing peaks at criticality. In the low density phase, only the delta function survives in the long-time limit.

As previously mentioned, the frontier between the low and strong noise regimes can be probed via the dynamical stability of the exponential phase. To do so, we study the evolution of the total mass in the system $M(t)=\int_{0}^{L} \rho(x, t) d x$, obtained by integrating eq. (2) with $g(\rho)=\sqrt{\rho}$ :

$$
\dot{M}=a M-b \int_{0}^{L} \rho^{2}(x, t) \mathrm{d} x+\Gamma_{0} \int_{0}^{L} \sqrt{\rho(x, t)} \eta(x, t) \mathrm{d} x
$$

The diffusion and advection terms have dropped out due to the boundary conditions. We approximate this by the following simplified Langevin equation 3 :

$$
\dot{M}=a M-\beta M^{2}+\Gamma_{0} \sqrt{M} \tilde{\eta}(t)
$$

where $\tilde{\eta}(t)$ is Gaussian white noise of unit variance. Both noise terms in (3) and (4) are gaussian, have the same mean and variance, and are therefore equivalent. The term $-\beta M^{2}$ is an approximation - we know from (3) that there must be saturation terms in the effective dynamics of $M(t)$, and retain only the lowest order in $M$. We believe this approximation to be reasonable as we only consider the low-density regime, and hence small mass. (In the high-density phase, the mass would be extensive with the system size and the approximation would break down.) The parameter $\beta$ contains the dependence on $v$ and $b$ but its form is not known exactly.

The dynamical stability of the exponential is not easily studied from (4) as the noise is multiplicative. We therefore recast it into an additive Langevin equation via the following change of variable $u=2 \sqrt{M} / \Gamma$. Using the Ito formula [14, (44) becomes

$$
\dot{u}=-\partial_{u} V_{\text {eff }}(u)+\eta ; \quad V_{\text {eff }}(u)=-\frac{a u^{2}}{4}+\frac{\beta \Gamma^{2} u^{4}}{32}+\frac{\log u}{2}
$$

The problem is now reduced to the diffusion of a particle in a potential $V_{\text {eff }}$ at temperature $T=1 / 2$. A steadystate solution is thus given by $P(u) \propto \exp \left[-2 V_{\text {eff }}(u)\right]$. One notes however that $P(u) \sim u^{-1}$ when $u \rightarrow 0$. The potential is thus not normalizable, which simply stresses that the low density phases are always absorbed as $t \rightarrow \infty$; the only normalizable steady-state solution is $P(M)=\delta(M)$. The shape of $V_{\text {eff }}$ nevertheless contains relevant information for the dynamics, as illustrated in Fig. 4a. When

\footnotetext{
${ }^{3} \mathrm{~A}$ similar approximation was used by Munoz in 3
}

$\Gamma_{0}<\Gamma_{c}=\frac{a}{\sqrt{\beta}}$, the effective potential has a local minimum corresponding to a potential well for positive $M$. The well lies above the global minimum at $M=0$ and corresponds to a metastable phase with finite mass: the exponential phase. Conversely, for $\Gamma_{0}>\Gamma_{c}$ there is no metastable state and the system falls directly into the absorbing state. $\Gamma_{c}$ thus corresponds to a spinodal point at which the exponential phase turns from metastable to unstable.
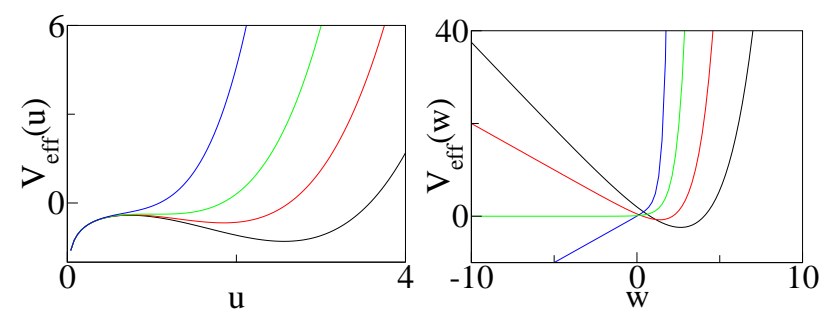

Figure 4: (a) Plot of the effective potential for $\eta \propto \sqrt{\rho}, a=2$, $\beta=0.5$. From top to bottom, we used $\Gamma_{0}=5.0 ; 2.8 ; 2.0 ; 1.5$ while $\Gamma_{c}=2 \sqrt{2}$. (b) Same for $\eta \propto \rho, a=2, \beta=0.5$. From top to bottom, we used $\Gamma_{0}=0.5 ; 1.0 ; 2.0 ; 4.0$ while $\Gamma_{c}=2$.

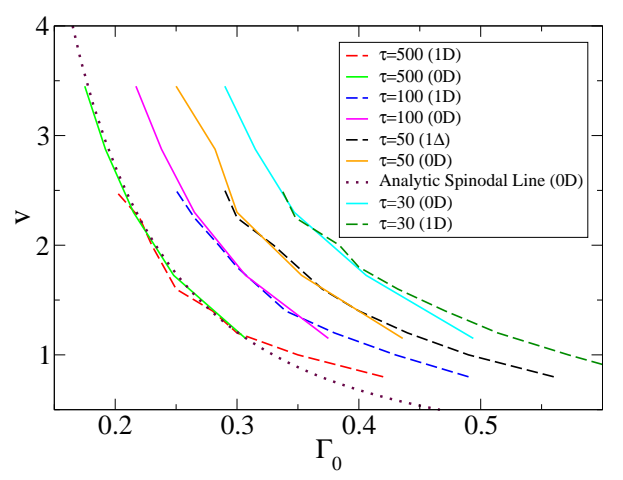

Figure 5: Contour lines in the $\left(v, \Gamma_{0}\right)$ plane for which $\tau$ equals, from left to right, 500, 100, 50 and 30 . In order to plot the contour lines of the 0D model, we use $\beta=2.3 v$ (see below). The analytical prediction for the spinodal line is also plotted, and closely matches the curves for which $\tau=500$.

To construct the spinodal line $\Gamma_{c}(v)$ from the 0D model, we try to relate $\beta$ to $v$ (the dependence on $b$ is not relevant since $b$ is constant throughout our study). To do so, we compare the mean time $\tau$ taken, for both the system and its $0 \mathrm{D}$ approximation, to reach the absorbing state. We compute numerically a set of contour lines for $\tau$ in the planes $\left(v, \Gamma_{0}\right)$ and $\left(\beta, \Gamma_{0}\right)$, corresponding to the $1 \mathrm{D}$ and $0 \mathrm{D}$ systems respectively (see Fig. 5). Strikingly, simply setting $\beta \simeq 2.3 v$ suffices to make the two sets of contour lines overlap. This strongly supports the validity of the $0 \mathrm{D}$ model and hence validates our interpretation of the frontier between low and strong noise regimes as a dynamical phase transition. We see on Fig. 5 that the theoretical prediction for the spinodal line corresponds to a

\footnotetext{
${ }^{4}$ For the 0D model, this mean first passage problem can be solved exactly up to a numerical integration 15]
} 
mean first passage time (MFPT) to absorption of $\tau=500$. In simulations, we indeed observe long-lived exponential profiles when the MFPT to absorption is larger than 500 whereas they are barely seen otherwise. The simulation data corresponding to $\tau=500$ was thus used to pinpoint the spinodal on Fig. 1.

We now turn to the investigation of the dependence of the phase diagram on the type of noise used in eq. (2), by considering $g(\rho)=\rho$. Without advection $(v=0)$, this model was studied in 3 , 16, 17] and it was shown that the system can be mapped onto the KPZ equation, by means of a Cole-Hopf transformation. As a consequence, the absorbing state phase transition at $v=0$ is continuous, but does not belong to the DP universality class. For instance, holding $\Gamma_{0}$ constant and varying $a$ close to criticality yields $\langle\rho\rangle \sim\left(a-a_{c}\right)^{\beta}$ with $\beta=1.5$, whereas $\beta \simeq 0.22$ in DP [11]. In our system, upon switching from the square root to the linear noise, the phase diagram is dramatically altered (Fig. 6).

First, the exponential profile is now completely stable for small $\Gamma_{0}$ and the transition between low and strong noise is a true phase transition. To understand why, we rely again on a $0 \mathrm{D}$ model, obtained by replacing $\sqrt{M}$ by $M$ in the noise term of eq. (4). We this time consider the change of variable $w=\Gamma^{-1} \log M$ to obtain an additive Langevin equation. Using the Ito formula [14 the corresponding equation reads

$$
\dot{w}=-\partial_{w} V_{\text {eff }}(w)+\eta ; \quad V_{\text {eff }}(w)=\left(\frac{\Gamma}{2}-\frac{a}{\Gamma}\right) w+\frac{b}{\Gamma^{2}} \mathrm{e}^{\Gamma w}
$$

and the putative steady-state is given by $P(w) \propto$ $\exp \left[-2 V_{\text {eff }}(w)\right]$. For $\Gamma<\sqrt{2 a}$, this is normalizable and the system is thus not absorbed, there is a proper normalizable steady-state distribution with $M \neq 0$. For $\Gamma \geq \sqrt{2 a}$, $\exp \left[-2 V_{\text {eff }}(w)\right]$ is not normalizable and the steady-state distribution once again corresponds to a delta function, the system will be absorbed. Considering now the stability of the exponential profile, we see that the effective potential has a single minimum which switches from $w=-\infty$ $(M=0)$ to finite $w$ depending on the sign of the first term. The transition point $\Gamma_{c}=\sqrt{2 a}$ corresponds to the normalization criterion, the spinodal line has become a true phase transition. Note that the critical line $\Gamma_{c}(a, \beta)=\sqrt{2 a}$ is now independent of $\beta$ and hence of the velocity. Numerically, we find that, for $a=1 / 2$, the transition line is almost vertical and very close to $\Gamma_{c}(v)=1$. This close agreement with our predictions is reinforced by the absence of any fitting parameter. As we approach the high-density phase, the mass of the exponential profile increases, and the slight bend of the transition line is presumably due to non-linear terms beyond the quadratic one, neglected in the $0 \mathrm{D}$ approximation. In the low noise regime, for $\Gamma_{0}<\Gamma_{c}$, the exponential state is now stable rather than metastable and hence constitutes a true phase.

Beyond the transformation of the dynamical transition into a true phase transiton, the transition between the high- and low-density phases (whether exponential or ab-

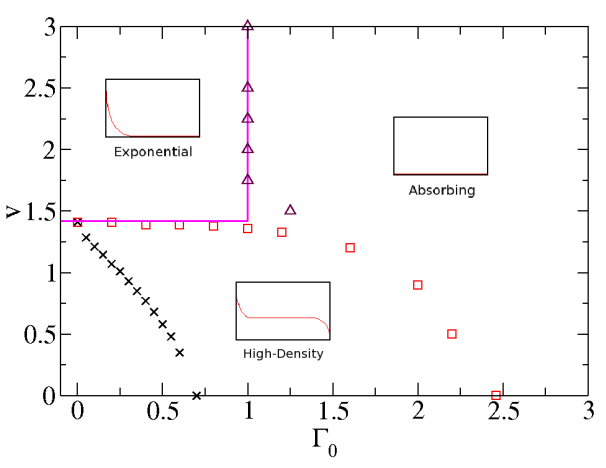

Figure 6: Phase diagram for $\eta \propto \rho(a=0.5)$. The red squares correspond to the transition between high- and low-density phases. Within the low-density region, there is now a second true phase transition between low and strong noise, indicated by the maroon triangles. For comparison, we include the phase boundary of the square root noise case (black crosses). The horizontal magenta line corresponds to the zero-noise transition point $v_{c} \simeq 1.41$ whereas the vertical one is the theoretical prediction from the $0 \mathrm{D}$ model.

sorbing) is also changed significantly. To pinpoint this critical line, we proceed numerically as before. The model is now easier to simulate since close to the transition, when $\rho \ll a / b$, the fluctuations remain of order $\rho$ and are much smaller than in the previous case, where they scaled as $\sqrt{\rho}$. Rare events leading to absorption are unlikely and Dickman's algorithm works very well, in particular discretization of the density is unnecessary. Our results suggest that for small noise, fluctuations are irrelevant and one recovers a transition identical to one of the deterministic limit - see Fig. 7. Indeed, numerically the transition line between exponential and high-density regimes is independent of $\Gamma_{0}$-hence horizontal - with $v_{c} \simeq 1.41$ as in the noiseless limit. Note that this transition is discontinuous and the band is stationary - in sharp contrast with the $\sqrt{\rho}$ noise where it performed a random walk at criticality. In the large noise case, where the stable state is absorbing rather than exponential, our simulations are consistent with a continuous transition (see Fig. 8) and more data would be needed to discriminate it from the $v=0$ limiting case.

In conclusion, we have studied the effect of advection and the role of noise in the context of continuous absorbing phase transitions, and directed percolation in particular. Our main result is that as soon as an advective velocity is added, the transition between the low- and high-density phases becomes discontinuous and therefore is no longer in the DP universality class. It is important to note that, in line with other terms in DP, the advection term is a nonequilibrium term, as it is related to the transport rather than to the thermodynamic properties of the system. Furthermore, we have illustrated that the type of noise used to model non-equilibrium phase transitions can control the kind of transitions observed. For instance, switching from square root to linear noise in our 


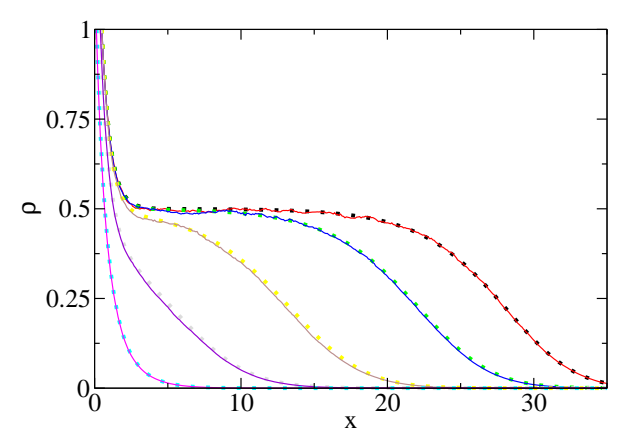

Figure 7: Plots of the steady state density profiles for the linear noise $\left(\Gamma_{0}=0.1\right.$, solid lines) and noiseless $\left(\Gamma_{0}=0\right.$, dotted lines) cases, for $L=40$ and values of $v$, from right to left, 1.30, 1.36, $1.39,1.40$ and 1.41 .

system transformed a dynamical phase transition into a true phase transition, stabilizing a previously metastable state. In addition to this, it appears that a large portion of the critical line has changed from discontinuous to continuous, although more extensive simulations would be required to confirm this. This important quantitative and qualitative difference highlights once again 3 that care should be exercised when deriving fluctuating hydrodynamic equations for non-equilibrium models, as the very form of the noise, which is sometimes overlooked, may drive unexpected changes in the physics of the system.

We close with a brief mention of a possible application of our results to experiments with microbial systems. In population dynamics, Eq. 2 at zero or small noise has been used to model bacterial sedimentation in pipettes [8], plankton sinking in oceans [18] (although in that work the logistic growth law was substituted by a non-local forcing term), and the persistence of populations of aquatic organisms in rivers [19] (i.e. the problem of how organisms can resist being swept upstream by advection from the flows in the rivers they inhabit). In all of these cases, experiments suggest that the Fisher equation is a good, although approximate, starting point to study these systems in the absence of advection: therefore the regime of interest in our calculations is the small noise one where a front can still be identified. Our results suggest that in this situation, being at or (for finite size systems) close to criticality brings with it a giant increase in fluctuations, and as a result we would predict that the system under these conditions would be unable to reach a steady state: the Fisher wavefront would indefinitely perform a random walk instead. It would be interesting to see whether controlled experiments in bacterial colonies subject to, e.g., uniform flow in microfluidic devices may be designed which give a transition between a persistent phase in which the Fisher wave moves faster than the flow to another one in which the bacteria are swept away by the moving fluid. If this is the case, our results suggest that it would be extremely interesting to closely monitor the behaviour of these experiments close to this transition.

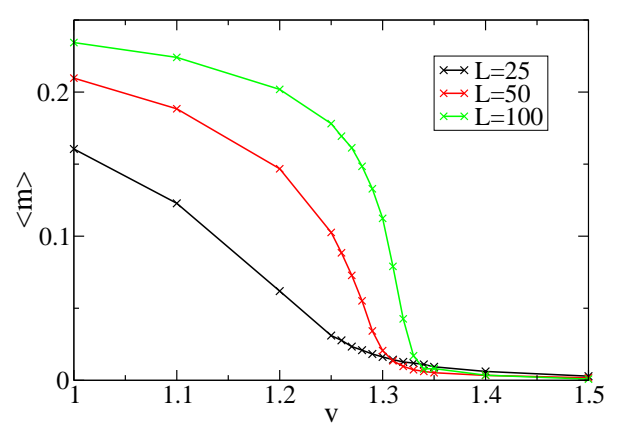

Figure 8: Plots of the average order parameter $\langle m\rangle$ as a function of $v$, for $\Gamma_{0}=1.25$ in the linear noise case, for three different system sizes (see legend). This figure is consistent with a continuous transition.

This work has made use of the resources provided by the Edinburgh Compute and Data Facility (ECDF). ( http://www.ecdf.ed.ac.uk/). The ECDF is partially supported by the eDIKT initiative ( http://www.edikt.org.uk). CBF is funded by EPSRC and JT acknowledges funding from EPSRC grant EP/030173.

References

[1] H. Hinrichsen, Adv. Phys. 49, 815 (2000)

[2] H.K. Janssen, Z. Phys. B 42, 151-154 (1981)

[3] M. A. Munoz Phys. Rev. E 57, 2, (1998)

[4] P. Grassberger, Z. Phys. B 47, 365 (1982)

[5] W. van Saarloos, Phys. Rep. 386, 29 (2003).

[6] K. A. Dahmen, D. R. Nelson, N. M. Shnerb, J. Math. Biol. 41, 1 (2000).

[7] D. Mukamel in Soft and Fragile Matter IoP Publishing, Bristol (2000)

[8] C. Barrett-Freeman, M. R. Evans, D. Marenduzzo, W. C. K. Poon, Phys. Rev. Lett. 101, 100602 (2008).

[9] B. Derrida, D. Simon, Europhys. Lett, 7860006 (2007); J. Stat. Phys., 131203 (2008).

[10] R. M. May, American Naturalist 107, 621 (1973).

[11] R. Dickman, Phys. Rev. E, 50, 6, (1994)

[12] I. Dornic, H. Chate, M.A. Munoz, Phys. Rev. Lett., 94, 100601 (2005).

[13] P. Grassberger, Comput. Phys. Commun. 147, 64 (2002).

[14] B. Oksendal, Stochastic Differential Equations, (Springer Berlin, 1998)

[15] F.T. Arecchi, A. Politi, L. Ulivi, Il Nuovo Cimento, 71B, 119 (1982).

[16] G. Grinstein, M. A. Munoz, Y, Tu, Phys. Rev. Lett. 76, 4376 (1996).

[17] Y. Tu, G. Grinstein, M. A. Munoz, Phys. Rev. Lett. 78, 274 (1997).

[18] J. Huisman, M. Arrayas, U. Ebert, B. Sommeijer, The American Naturalist 159, 245 (2002).

[19] D. C. Speirs, W. S. C. Gurney, Ecology 82, 1219 (2001). 
(a)

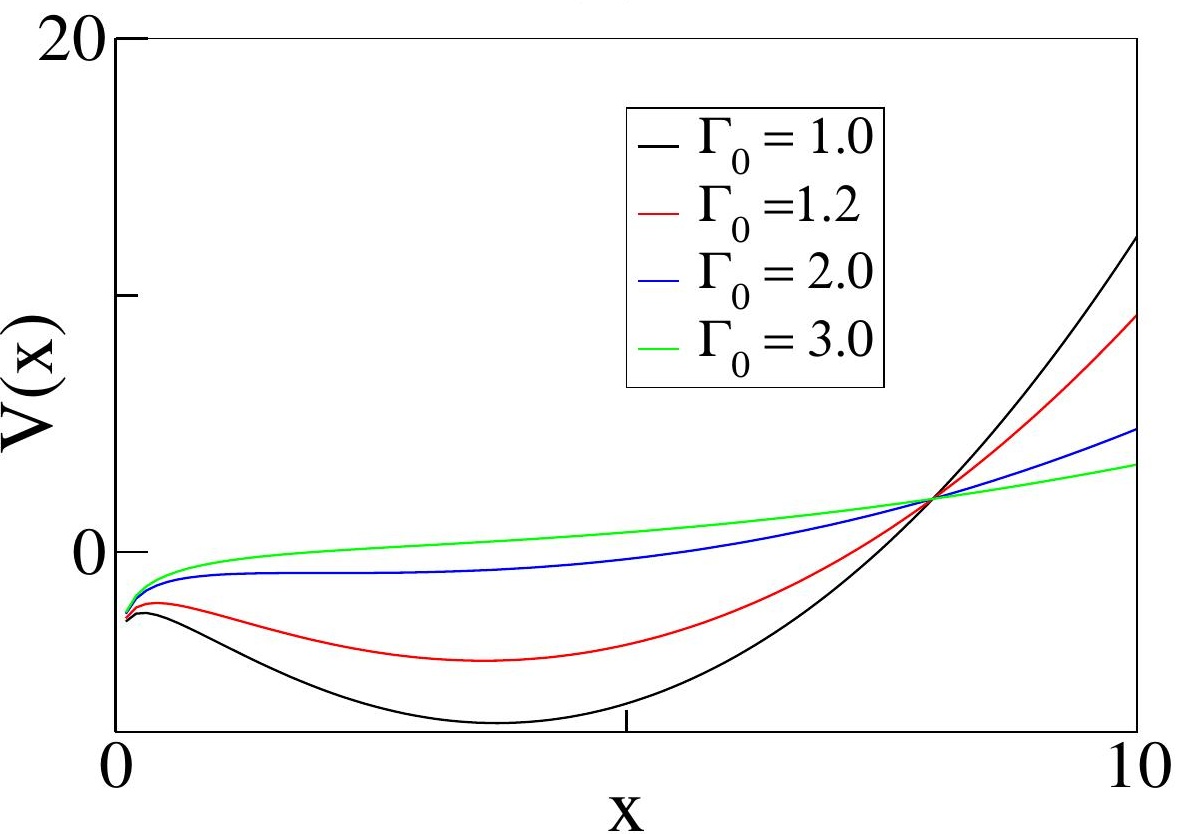

(b)

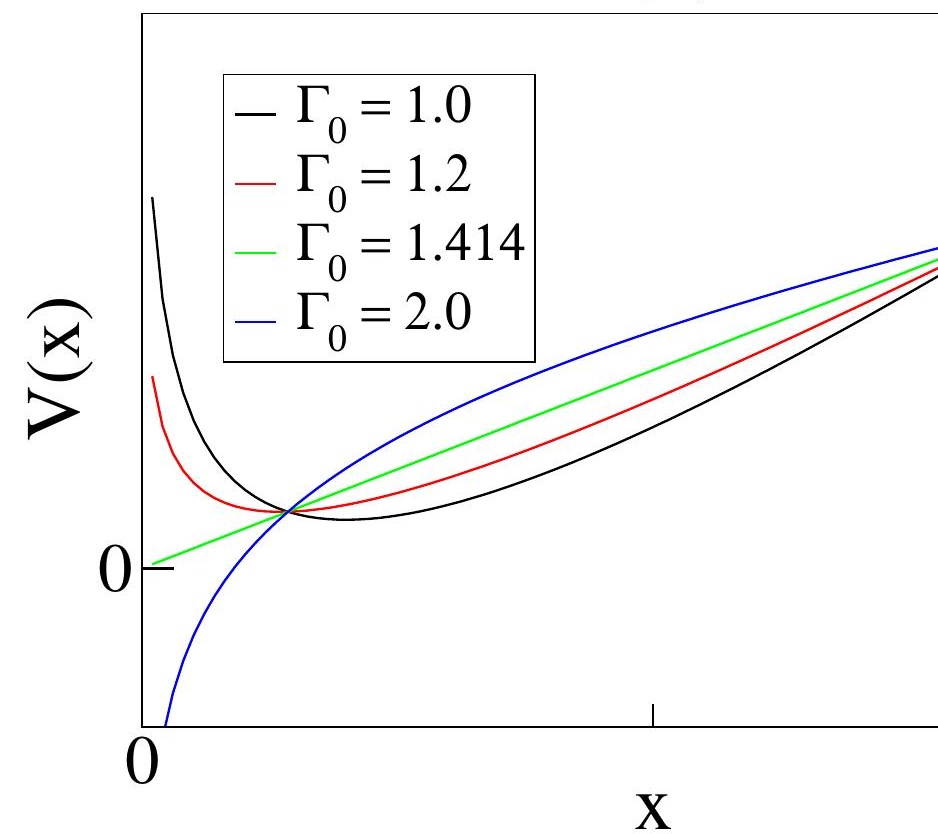

